\title{
President's Corner (Mitteilungen der SPS)
}

Schmerz 2020 · 34:196

https://doi.org/10.1007/s00482-020-

00457-x

(c) Springer Medizin Verlag GmbH, ein Teil von Springer Nature 2020

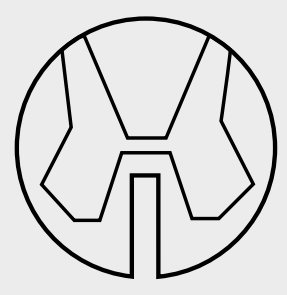

\section{Swiss Pain Society}

SPS Zentralsekretariat

c/o Pomcanys Marketing AG

Aargauerstrasse 250

8048 Zürich

Tel. +41444961016

E-Mail: info@swisspainsociety.ch

Web: www.pain.ch

Redaktion

PD Dr. med. Konrad Maurer, SPS Präsident (v. i. S. d. P.)
Liebe Mitglieder der Swiss Pain Society

\section{0-jähriges Jubiläum}

2020 ist ein besonderes Jahr für uns. Die Gesellschaft feiert 30 Jahre Studium des Schmerzes.

$\mathrm{Zu}$ diesem Anlass haben wir vor kurzem unsere neue Website vorgestellt. Frisch, schlank, übersichtlich und reich an Informationen über unsere Gesellschaft, sowie verschiedener Aktivitäten an denen man sich beteiligen kann. Statten auch Sie www. swisspainsociety.ch einen Besuch ab (• Abb. 1)!

An unserem geplanten Jahreskongress vom 1.-2. Oktober 2020 in Bern möchten wir zusammen mit der Schweizerischen Kopfwehgesellschaft das langjährige Bestehen beider Gesellschaften feiern. SKG - 25 Jahre | SPS - 30 Jahre. Feiern Sie mit und reservieren Sie sich schon jetzt den 1. und 2. Oktober 2020.

Auch unser Schmerzkurs hat dieses Jahr einen Jahrestag. Bereits 15 Jahre werden an diesem Kurs in Zürich Fachpersonen aus verschiedensten Disziplinen zum Thema Schmerz ausgebildet.

\section{Presseerklärung zu ICD-11}

Wie bereits angekündigt haben wir zusammen mit der Österreichischen sowie der Deutschen Schmerzgesellschaft eine gemeinsame Presserklärung zur Veröffentlichung von ICD-11 durch die WHO veröffentlicht. Dies ist ein Meilenstein für die Schmerzmedizin, denn erstmals werden chronische Schmerzen als eigenständige Krankheit erkannt. Mit einer gemeinsamen Erklärung der drei deutschsprachigen Schmerzgesellschaften haben wir eine etwas bessere Chance, von der Politik oder den öffentlichen Medien gehört zu werden.

Ich freue mich dieses Jahr mit Ihnen dankbar zurück zu blicken und den bisherigen Erfolg zu feiern! Auf weitere zielgerichtete Jahre im gemeinsamen Einsatz in der Schmerztherapie.

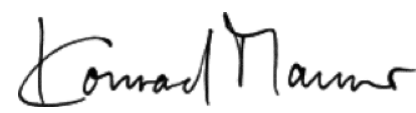

PD Dr. med. Konrad Maurer SPS Präsident (2017-2020)

\section{Wichtige SPS-Events 2020/2021}

(Detaillierte Informationen zu jeder Veranstaltung finden Sie auf unserer Website www.swisspainsociety.ch)

\section{SPS Schmerzkurs 2020}

Der multidisziplinäre Schmerzkurs findet zum 15. Mal in $5 \mathrm{Ab}-$ schnitten von März bis Juni 2020 im Sorell Hotel Zürichberg in Zürich statt.

\section{SPS Jahreskongress 2020}

Donnerstag und Freitag, 1.2. Oktober 2020 in Bern gemeinsam mit der Schweizerischen Kopfwehgesellschaft SKG

\section{SPS Jahreskongress 2021}

Freitag, 10. September 2021 in Lausanne gemeinsam mit Chiro-Suisse

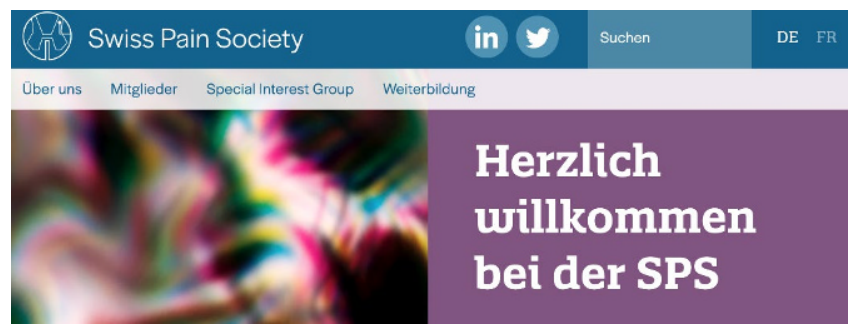

Abb. $1 \Delta$ neue Website der Swiss Pain Society 